\title{
Advanced Glycation End Products and its Soluble Receptors in the Pathogenesis of Thoracic Aortic Aneurysm
}

\author{
Kailash Prasad, MBBS (Hons), MD, PhD, DSc, FRCPC, FACC, FICA ${ }^{1 *}$, Abdullah Sarkar²,3, \\ Mohammad A. Zafar, MBBS ${ }^{2}$, Ahmed Shoker, MD, FRCP ${ }^{4}$, Hamdi El Moselhi, PhD ${ }^{4}$, \\ Maryann Tranquilli, RN², Bulat A. Ziganshin, MD ${ }^{2,5}$, John A. Elefteriades, MD² \\ ${ }^{1}$ Department of Physiology, College of Medicine, University of Saskatchewan, Saskatoon, Saskatchewan, Canada \\ ${ }^{2}$ Aortic Institute at Yale-New Haven, Yale University School of Medicine, Connecticut, USA \\ ${ }^{3}$ College of Medicine, Alfaisal University, Ryadh, Saudi Arabia \\ ${ }^{4}$ Department of Medicine, Royal University Hospital, Saskatoon, Saskatchewan, Canada \\ ${ }^{5}$ Department of Surgical Diseases \#2, Kazan State Medical University, Kazan, Russia
}

\begin{abstract}
Background: Matrix metalloproteinases (MMPs) have been implicated in the pathogenesis of thoracic aortic aneurysms (TAAs). Cytokines [Interleukin (IL)-I $\beta$, IL-2, IL-6, and TNF-a)] increase the expression of MMP-2 and -3 . Advanced glycation end products (AGEs) interact with cell receptors to increase the release of cytokines. Circulating soluble receptors for AGEs (sRAGE) and endogenous secretory RAGE (esRAGE) compete with membrane bound RAGE for binding with AGEs and reduce the production of cytokines. It is hypothesized that low levels of serum sRAGE and esRAGE and high levels of AGEs, AGEs/sRAGE, and AGEs/esRAGE would increase the levels of cytokines that would increase the levels MMPs, thus contributing to the formation of TAAs.

Methods: The study population was composed of 17 control subjects and 20 patients with TAA. Blood samples were collected for measurement of serum sRAGE, esRAGE, AGEs, cytokines, and MMPs. AGEs, SRAGE, and esRAGE were measured using ELISA kits, whereas the remaining parameters were measured using the Luminex Multi-Analyte system.

Results: The levels of SRAGE were lower, while the levels of AGEs, AGEs/sRAGE, AGEs/esRAGE, cytokines

Published by Science International Corp. ISSN 2325-4637

and MMPs were higher in patients with TAA compared to controls. The levels of SRAGE were inversely correlated with cytokines and MMPs, while AGEs, AGEs/sRAGE and AGEs/esRAGE were positively correlated with cytokines and MMPs. Cytokines were positively correlated with MMPs.

Conclusions: The data suggest that the AGE-RAGE axis may be involved in the pathogenesis of TAA and that low levels of sRAGE and high levels of AGEs, AGEs/ SRAGE, and AGEs/esRAGE are risk factors for TAA.

Copyright $\odot 2016$ Science International Corp.

\section{Key Words}

Advanced glycation end products - Soluble RAGE • Cytokines - Matrix metalloproteinase - Aortic aneurysm • Thoracic aortic aneurysm

\section{Introduction}

The mechanism of development of thoracic aortic aneurysms (TAAs) is complex. The characteristic features of TAAs are destruction of collagen and elastin in media and adventitia, loss of smooth muscle cells with thinning of aortic wall, and transmural infiltration

* Corresponding Author:

Kailash Prasad, MBBS (Hons), MD, PhD, DSc

Department of Physiology, College of Medicine,

University of Saskatchewan,

107 Wiggins Road, Saskatoon, Saskatchewan, S7N 5E5, Canada

Tel: +1 306966 6539; Fax: +1 306966 4298; E-Mail: k.prasad@usask.ca 
of lymphocytes and macrophages. Matrix metalloproteinases (MMPs) -2 and -3, which have elastolytic and collagenolytic activities, have been implicated in the development of (aortic aneurysm) AA [1, 2]. Cytokines interleukin (IL)-1 $\beta$ and tumor necrosis factor-alpha (TNF- $\alpha$ ) increase the expression of MMP-2. IL-1 $\beta[3,4]$, IL-2 [5], IL-6 [6], and TNF- $\alpha$ [4] regulate the expression of MMP-2 and MMP-3.

Advanced glycation end products (AGEs) are a heterogeneous group of irreversible aducts resulting from nonenzymatic glycation and oxidation of proteins, nucleic acids and lipids $[7,8]$. AGEs interact with receptors for $A G E s$ (RAGE) and activate nuclear-factor kappa-B (NF-KB), increasing gene expression and releasing inflammatory cytokines (IL-1 $\beta$, IL-2, IL-6, and TNF-a) [8-10] and generating reactive oxygen species (ROS) $[8,11]$. There are two isoforms of c-truncated RAGE: total soluble RAGE (sRAGE) [12] and endogenous secretory RAGE (esRAGE) [13]. Both sRAGE and esRAGE act as a decoy for RAGE ligands and compete with membrane-bound RAGE for ligand binding [14], thus reducing the production of cytokines [3-6] and ROS [15]. Earlier, based on the literature, we [16] had suggested that sRAGE plasma levels may differentiate patients with aortic disease from the general population.

It is hypothesized that low levels of sRAGE and esRAGE and high levels of AGEs, AGEs/sRAGE, and AGEs/esRAGE would increase the levels of cytokines, which in turn would increase the levels of MMPs, resulting in the formation of aortic aneurysms. The specific objectives are to determine whether serum levels of sRAGE and esRAGE are lower, and AGEs, AGEs/sRAGE, AGEs/esRAGE, cytokines (IL-1 $\beta$, IL-2, IL-6, TNF- $\alpha$ ), and MMPs (MMP-2 and MMP-3) are higher in patients with TAA compared to control subjects. The other objectives are to determine if SRAGE and esRAGE are negatively correlated with cytokines and MMPs; if AGEs, AGEs/sRAGE, and AGEs/esRAGE are positively correlated with cytokines and MMPs; and if cytokines are positively correlated with MMPs.

\section{Methods}

\section{Study Population}

The study population was composed of 17 control and 20 patients with TAA. The control subjects were selected at the Royal University Hospital, University of Saskatchewan,
Saskatoon, Canada. The following selection criteria for control subjects were used. They were in the age group of 35 to 50 years, nonobese, normotensive, nonsmokers, and had no history of angina, coronary artery disease, or diabetes. The patients with TAA were selected from Aortic Institute, Yale-New Haven Hospital, Yale University School of Medicine, (New Haven, Connecticut, USA) during the period of September 2011 to May 2012. The demographics and clinical characteristics of the patients with TAAs are shown in Table 1. The study protocol was approved by the Human Investigations Committee at Yale University, School of Medicine, New Haven, Connecticut, and the Ethics Committee for Human Studies at the University of Saskatchewan and by the Saskatoon Health Region. Written informed consent was obtained from control subjects and patients with TAA. TAA in figures has been represented as AA.

\section{Measurements of Biochemical Parameters}

Five milliliters of arterial/venous blood samples were collected from control subjects and intra-operatively from all patients undergoing surgery of thoracic aorta before heparin administration in a vacutainer without anticoagulant for measurement of serum sRAGE, esRAGE, AGEs, IL-I $\beta, I L-2$, IL-6, TNF-a, MMP-2, and MMP-3. Blood samples were immediately refrigerated at $4^{\circ} \mathrm{C}$ for 3 hours before centrifugation at $3000 \mathrm{rpm}$ for 10 minutes at $4^{\circ} \mathrm{C}$. The serum (supernatant) was collected and transferred into Eppendorf tubes and stored at $-80^{\circ} \mathrm{C}$ until used for analysis. AGE levels in the serum were measured using a human AGE-enzyme-linked immunoassay (ELISA) kit (BioPCR, Beijing Zhonghao Shidai Co. Ltd. China). Serum levels of sRAGE and esRAGE were measured using the commercially available ELISA kit (R\&D systems, Minneapolis, Minnesota, USA). IL-1 $\beta$, IL-2, IL-6, TNF- $\alpha$, MMP-2, and MMP-3 were measured using Luminex Multi-Analyte Profiling System (Luminex, Austin Texas, USA, Bio-Rad) an instrument that measures multiple analytes simultaneously in one sample $[17,18]$

\section{Statistical Analysis}

The data are reported as the mean \pm SE. The data between the two groups were compared using a 2-tailed unpaired Student's $t$ test. Single linear univariate correlations (Pearson's correlation cofficients) were performed to evaluate the relationship between AGEs, sRAGE, or esRAGE, and cytokines and MMPs, between AGEs, AGEs/sRAGE, or AGEs/esRAGE, and cytokines, and between cytokines and MMPs. A p value of less than 0.05 was considered significant.

\section{Results}

Serum AGEs, sRAGE, and esRAGE

The serum levels of AGEs, sRAGE, and esRAGE in control subjects and in patients with TAA are summarized in Figure 1.

The serum levels of sRAGE were 1.43-fold lower $(997.5 \pm 84.06$ vs. $1425.0 \pm 106.63 \mathrm{pg} / \mathrm{ml})$, while levels 
Table 1. Demographics and clinical characteristics of the patients with aortic aneurysms.

\begin{tabular}{|c|c|}
\hline Parameter & Value (\%) \\
\hline Number of patients & 20 \\
\hline Age (years) \pm SD & $53.85 \pm 13.76$ \\
\hline Male & $15(75 \%)$ \\
\hline Female & $5(25 \%)$ \\
\hline \multicolumn{2}{|l|}{ Race: } \\
\hline African American & $1(5 \%)$ \\
\hline Hispanic & $1(5 \%)$ \\
\hline White & $18(90 \%)$ \\
\hline \multicolumn{2}{|l|}{ Risk factors/comorbidities: } \\
\hline Smokers (or history of smoking) & $6(30 \%)$ \\
\hline Body mass index & $34.29 \pm 0.25$ \\
\hline Obesity & $11(55 \%)$ \\
\hline Diabetes & $3(15 \%)$ \\
\hline Hypertension & $13(65 \%)$ \\
\hline Coronary artery disease & $3(15 \%)$ \\
\hline Marfan syndrome & $1(5 \%)$ \\
\hline Previous cardiac surgery & $3(15 \%)$ \\
\hline Previous aortic surgery & $1(5 \%)$ \\
\hline Positive family history & $5(25 \%)$ \\
\hline Mean size of ascending aorta, $\mathrm{cm}$ & $5.07 \pm 0.56$ \\
\hline
\end{tabular}

of AGEs were 6.3 folds higher $(20.06 \pm 3.69$ vs. $2.93 \pm$ $0.97 \mu \mathrm{g} / \mathrm{ml}$ ) in patients with TAA compared to controls. The values for esRAGE were not significantly different from each other $(0.372 \pm 0.0334$ vs. $0.326 \pm 0.0391$ $\mathrm{ng} / \mathrm{ml}$ : controls vs. patient.)

\section{AGEs/sRAGE, AGEs/esRAGE, MMP-2 and MMP-3}

The ratio of AGEs/sRAGE, AGEs/esRAGE, and serum levels of MMP-2 and -3 for control subjects and patients with TAA are summarized in Figure 2. The ratio of AGEs/sRAGE and AGEs/esRAGE were 10.4- and 8.18-fold higher, respectively, in patients with TAA compared to controls. The values for MMP-2 and -3 were $29.60 \%$ and $142.1 \%$ higher in patients with TAA compared to controls, respectively.

\section{Cytokines}

The serum levels of IL-I $\beta$, IL-2, IL- 6 , and TNF- $\alpha$ in patients with TAA and control subjects are summarized in Figure 3. The values of IL-I $\beta$, IL-2, IL- 6 , and TNF- $\alpha$ in control subjects were $0.587 \pm 0.10,0.402 \pm 0.066$, $1.375 \pm 0.363$, and $8.09 \pm 0.810 \mathrm{pg} / \mathrm{ml}$, respectively. The levels of IL-I $\beta, I L-2, I L-6$, and TNF- $\alpha$ in patients with AA were, respectively, 3.49-, 22.38-, 10.65-, and 3.35-fold higher than controls, but the values were significant only for IL-2 and IL-6.

Correlation of SRAGE with IL-I $\beta, I L-2, I L-6$, and TNF- $a$

Correlation data from 20 patients with TAA and 11 control subjects are summarized in Figure 4. The levels of serum sRAGE are negatively correlated with IL-I $\beta$, IL-2, and I- 6 but were significant only with IL-6. There was no correlation between sRAGE only and TNF-a.

\section{Correlation of AGEs with Cytokines}

The correlation between AGEs and cytokines is shown in Figure 5. The serum levels of AGEs are positively correlated with only IL-I $\beta$ and IL-6. Although there was a tendency for negative correlation of AGEs with IL-2 and TNF-a, the correlation was not significant.

\section{Correlation of AGEs/sRAGE with Cytokines}

The results are summarized in Figure 6 and Table 2. There was a positive correlation between AGEs/ sRAGE, and IL-I $\beta, I L-2, I L-6$, and TNF- $\alpha$ but the correlation was significant only between AGEs/sRAGE and IL-2.

\section{Correlation of esRAGE with Cytokines}

There was a tendency for a positive correlation of serum esRAGE with serum IL-I, IL-2, IL-6 and TNF-a, but the correlation was significant only between esRAGE and TNF-a (Table 2).

\section{Correlation of AGEs/esRAGE with Cytokines}

There was a tendency for a positive correlation between AGEs/esRAGE, and IL-I $\beta$ and TNF- $\alpha$, but the correlation was significant only between AGEs/ esRAGE and IL-2 and IL-6 (Table 2). 

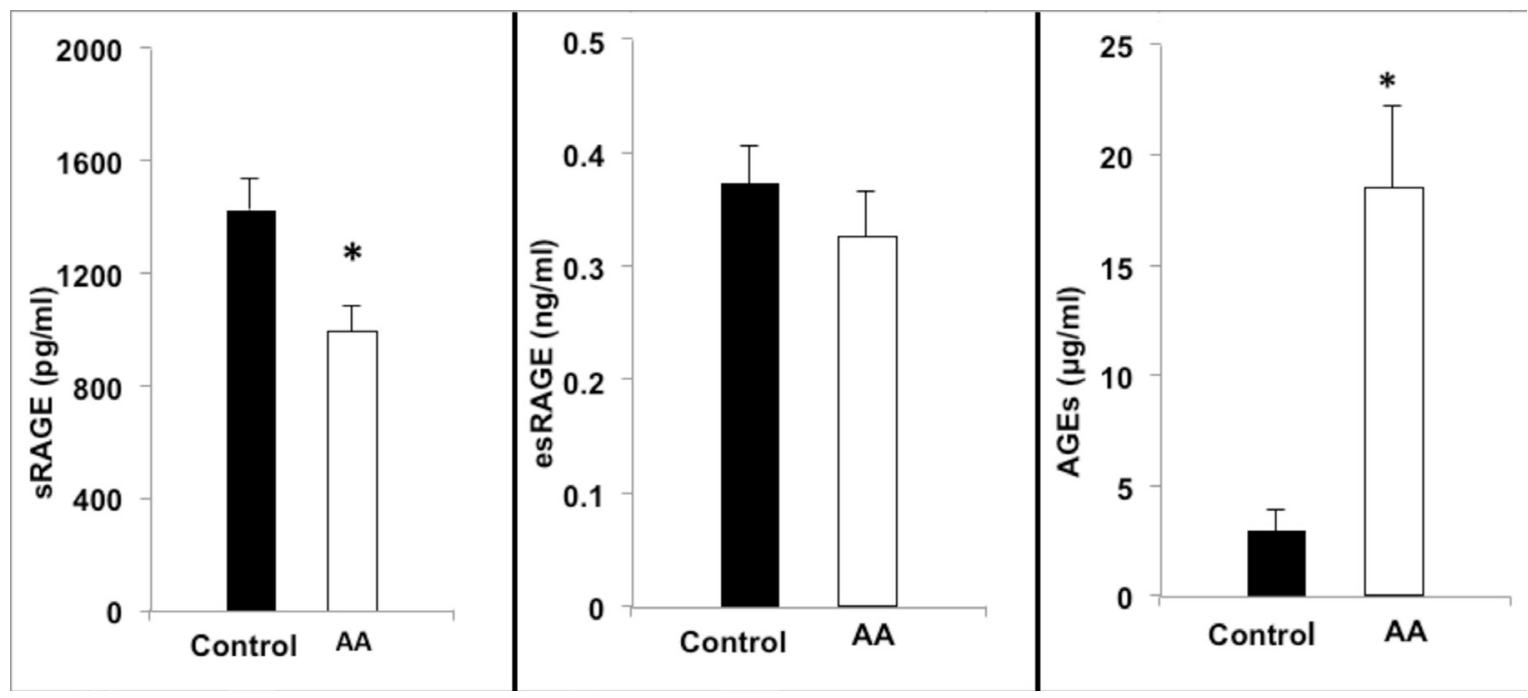

Figure 1. The serum levels of sRAGE, esRAGE, and AGEs in control subjects and in patients with ascending thoracic aortic aneurysms (AA). Results are expressed as the mean \pm SE. AGEs = advanced glycation end products; sRAGE $=$ soluble receptor for AGEs; esRAGE = endosecretory soluble receptor for AGEs. ${ }^{*} \mathrm{P}<0.05$; control vs. patients with $A A$.

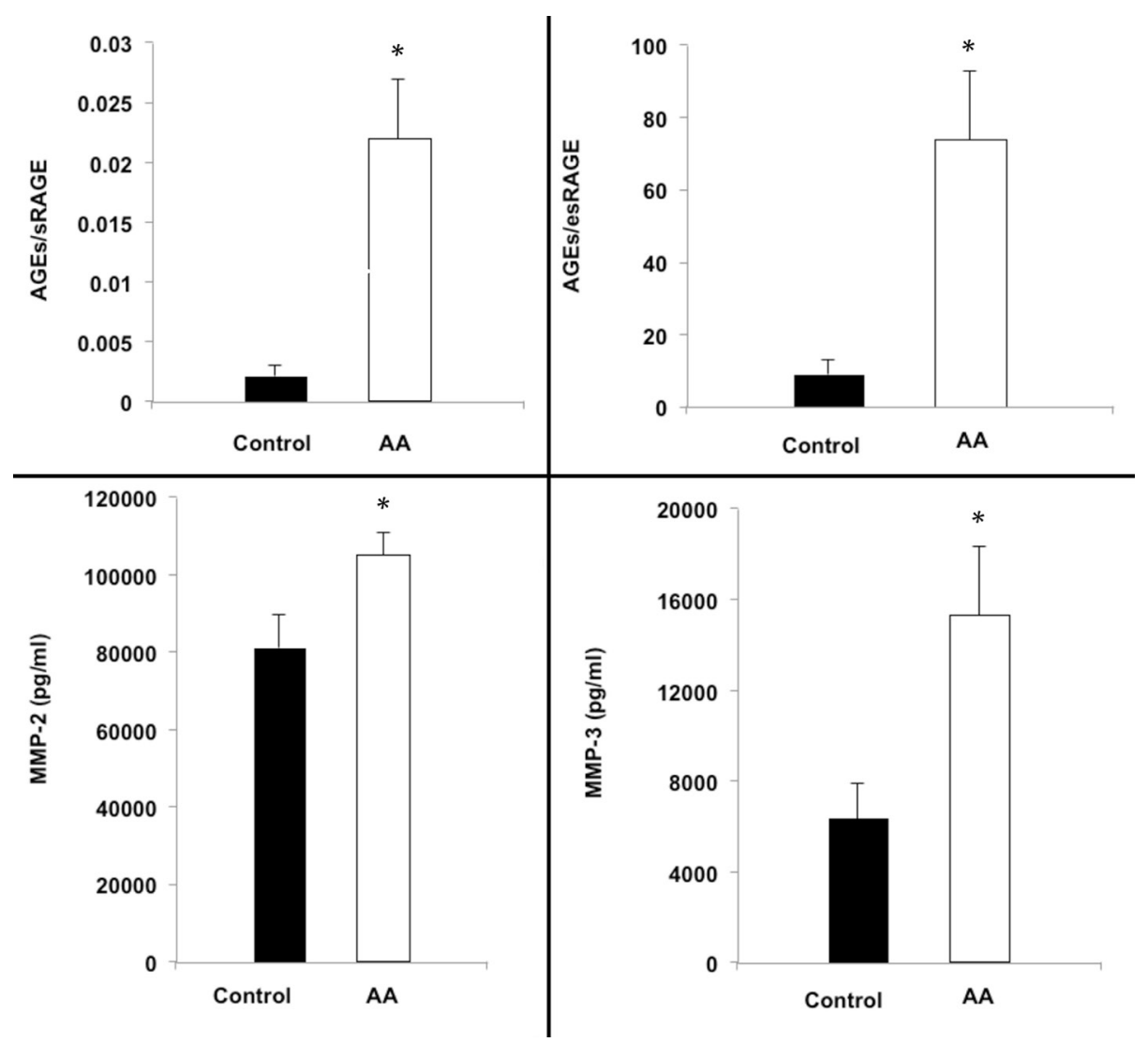

Figure 2. Ratios of AGEs/sRAGE, AGEs/esRAGE, and serum levels of MMP-2 and -3 in control subjects and patients with ascending thoracic aortic aneurysms (AA). Results are expressed as the mean \pm SE. AGEs = advanced glycation end products; sRAGE = soluble receptors for AGEs; esRAGE = endosecretory soluble receptors for AGEs; MMP = matrix metalloproteinase. *P $<0.05$, Control vs. patients with AA. 


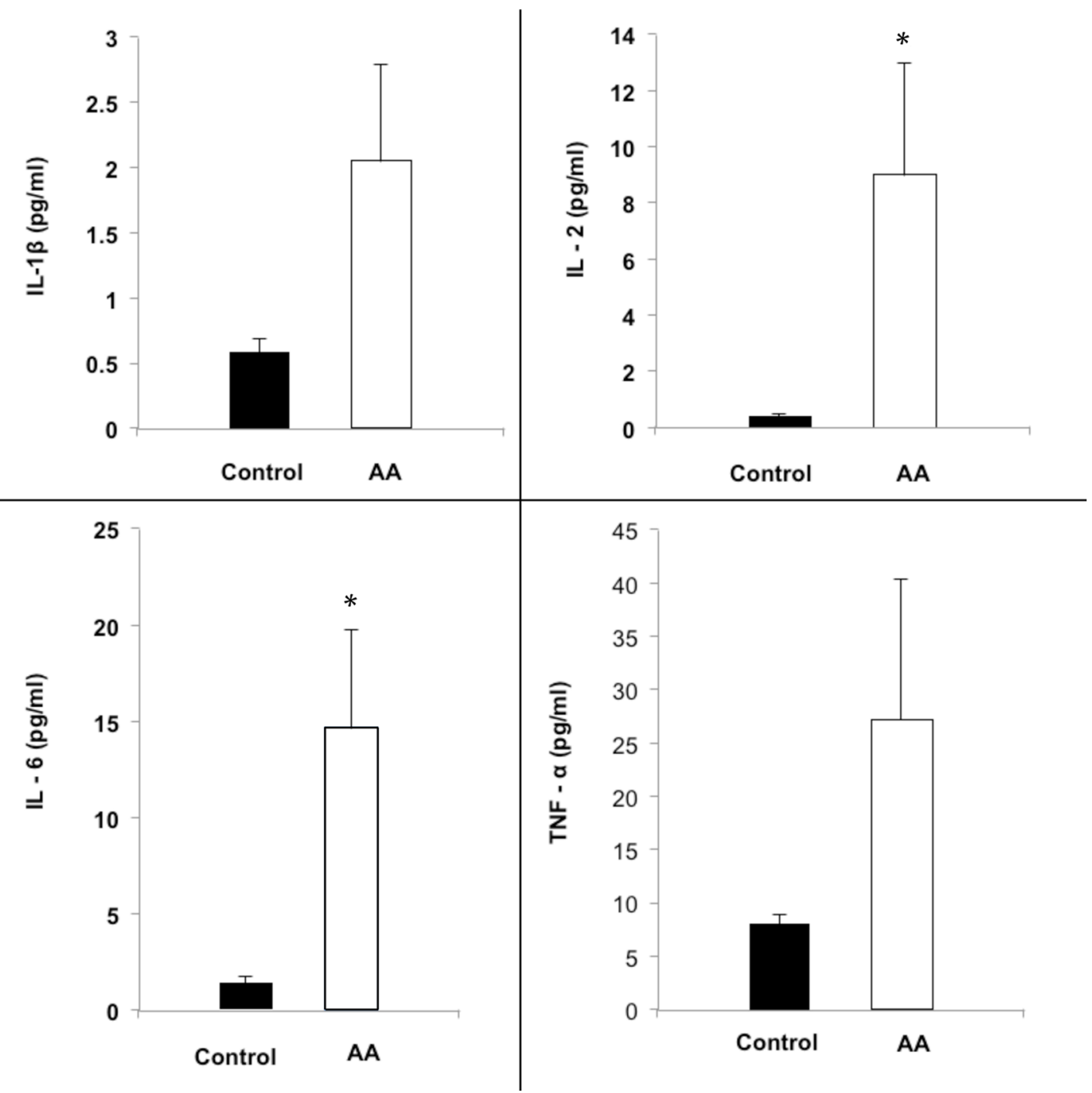

Figure 3. Serum levels of IL-I $\beta, I L-2, I L-6$, and TNF- $\alpha$ in control subjects and patients with TAA. Results are expressed as the mean $\pm S E$. IL = interleukin; TNF- $a=$ tumor necrosis factor-alpha. ${ }^{*} \mathrm{P}<0.05$; control vs. patients with $A A$.

Correlation of MMP-2 or MMP-3 with sRAGE, esRAGE, AGEs, AGEs/sRAGE, and AGEs/esRAGE

The results are summarized in Figure 6 and Table 2. There was a tendency for a positive correlation between SRAGE and MMP-2, and a negative correlation between SRAGE and MMP-3 (Figure 6). There was a positive correlation of esRAGE with MMP-2 and -3 but not significant (Table 2). There was a weak positive correlation of AGEs with MMP-2 and negative correlation with MMP-3 (Table 2) and weak correlation of AGEs/sRAGE with MMP-2 and -3 (Figure 6), and between AGEs/esRAGE and MMP-2 and -3 (Table 2).

\section{Correlation of Cytokines with MMP-2 and MMP-3}

The correlation between cytokines and MMP-2 and -3 are summarized in Figure 7 and Table 2. There was a positive correlation between MMP- 2 and IL-I $\beta$ (significant), IL-2, IL-6, and TNF- $\alpha$, and between MMP3 and IL-I $\beta$ (significant), IL-2, and TNF- $\alpha$.

\section{Discussion}

The data show that serum levels of sRAGE are lower, and the levels of AGEs, AGEs/sRAGE, and AGEs/ esRAGE are higher, in patients with TAA compared to 


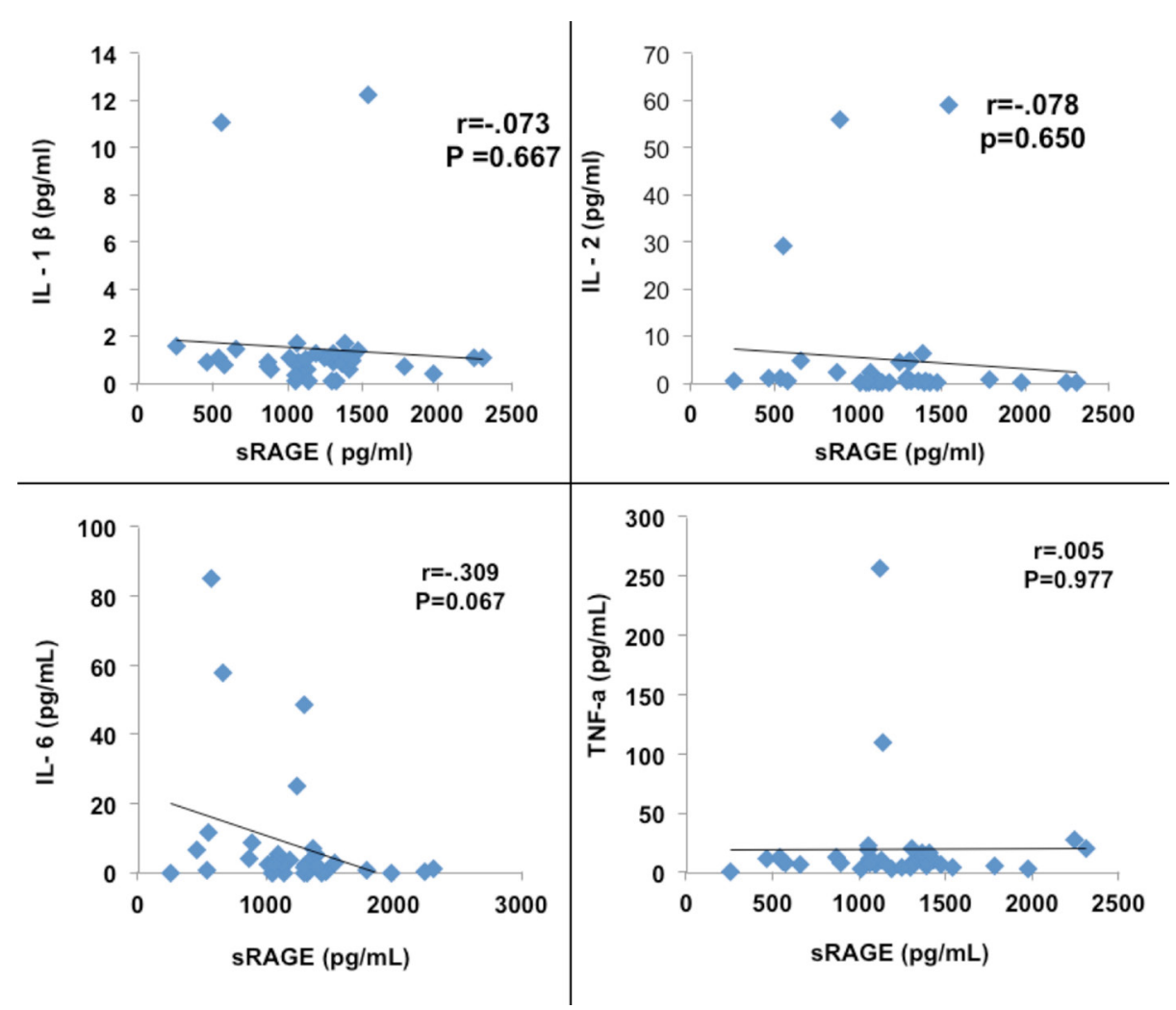

Figure 4. Correlation of sRAGE with IL-I, IL-2, IL-6, and TNF- $a$. IL = interleukin; TNF- $a=$ tumor necrosis factor-alpha; sRAGE = soluble receptor for advanced glycation end products.

control subjects. These are new findings. The levels of MMP-2, MMP-3, IL-I $\beta$, IL-2, IL- 6 , and TNF- $\alpha$ are higher in patients with TAA than in control subjects. Other investigators $[19,20]$ have also reported that the serum levels of IL- $\beta$, IL- 2 , IL- 6 , and TNF- $\alpha$ are elevated in patients with abdominal aortic aneurysm [19-21]. In the present study, although the levels of TNF-a were higher in patients with TAA compared to controls, they were not significantly different from each other. The elevated serum levels of MMP-2 and MMP-3 also have been reported in patients with abdominal aortic aneurysm and TAA [22-25].

In the present study there was a tendency for an inverse correlation between SRAGE and IL- $\beta$, IL- 6 , and IL-2. There was no significant inverse correlation between esRAGE and IL-I $\beta$, IL-2, and IL- 6 except with TNF- $\alpha$, which had a positive correlation with esRAGE. This positive correlation was not expected and the reason for this is not clear. There was a positive correlation between AGEs and cytokines (IL-I $\beta$ and IL-6) and between
AGEs/sRAGE and IL- $\beta$ or IL-6, but not with IL-2 or TNF- $a$. We had expected that there would be significant positive correlation between AGEs or AGEs/sRAGE and cytokines. It is possible that IL-I $\beta$ and IL- 6 are more frequently associated with the pathogenesis of TAA than are IL-2 and TNF-a.

There was a significant positive correlation of AGEs/esRAGE with IL-2 and IL-6 but not with TNF-a and IL-Iß. Again it is not known why there was no positive correlation between AGEs/esRAGE and IL-I $\beta$ and between AGES/esRAGE and TNF-a. May be that the sample size was too small to evoke all correlations that may eventually prove positive.

It was expected that low sRAGE and esRAGE would have negative correlation with MMP-2 and MMP-3, and AGEs/sRAGE would have positive correlation with MMP-2 and -3 . However, it was found that only SRAGE have negative correlations with MMP-2 and AGEs/sRAGE have weak positive correlations with MMP-2 and -3 . 

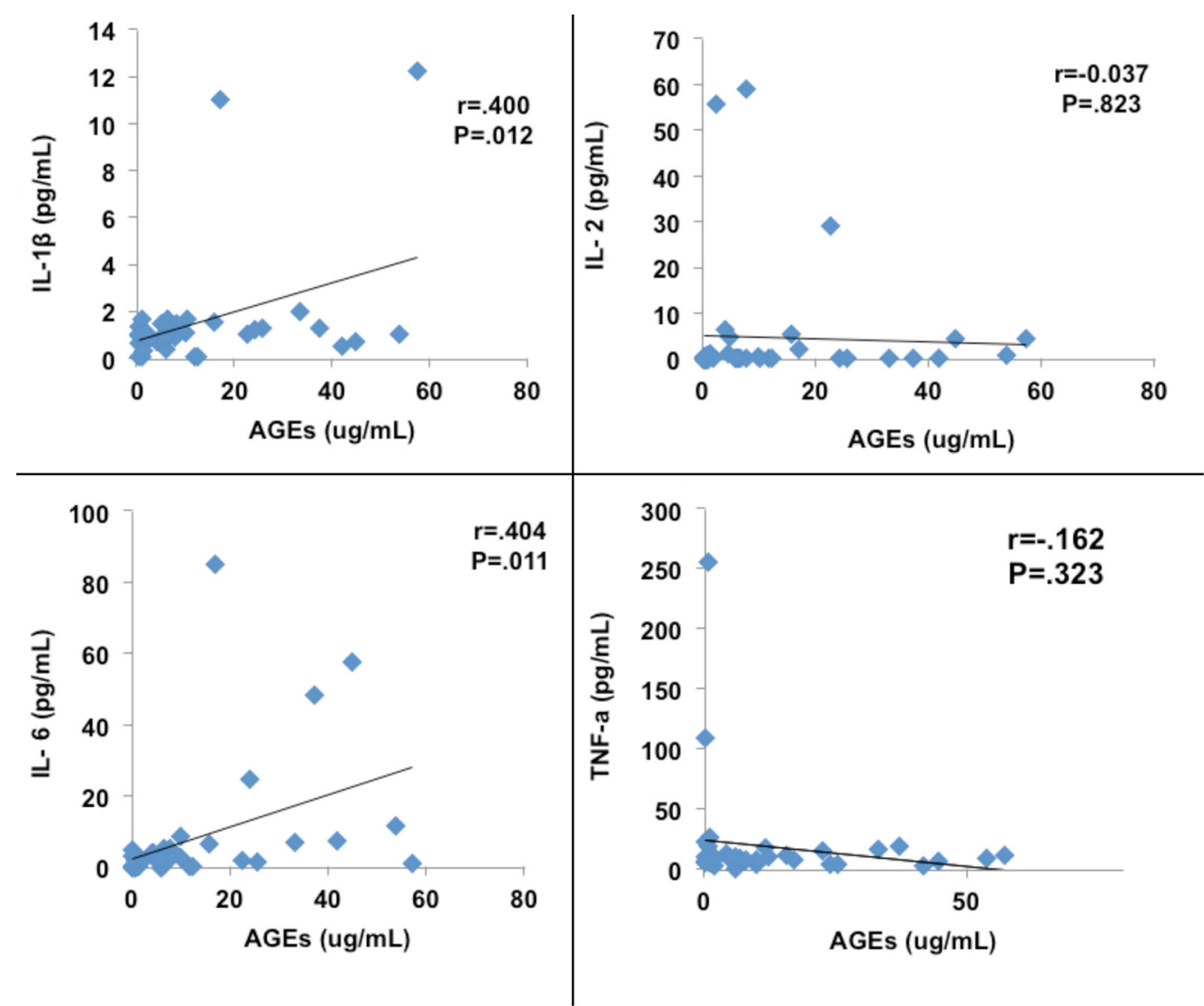

Figure 5. Correlation of AGEs with IL-I $\beta, I L-2, I L-6$, and TNF-a. AGEs = advanced glycation end products; IL = interleukin, TNF- $a=$ tumor necrosis factor-alpha.

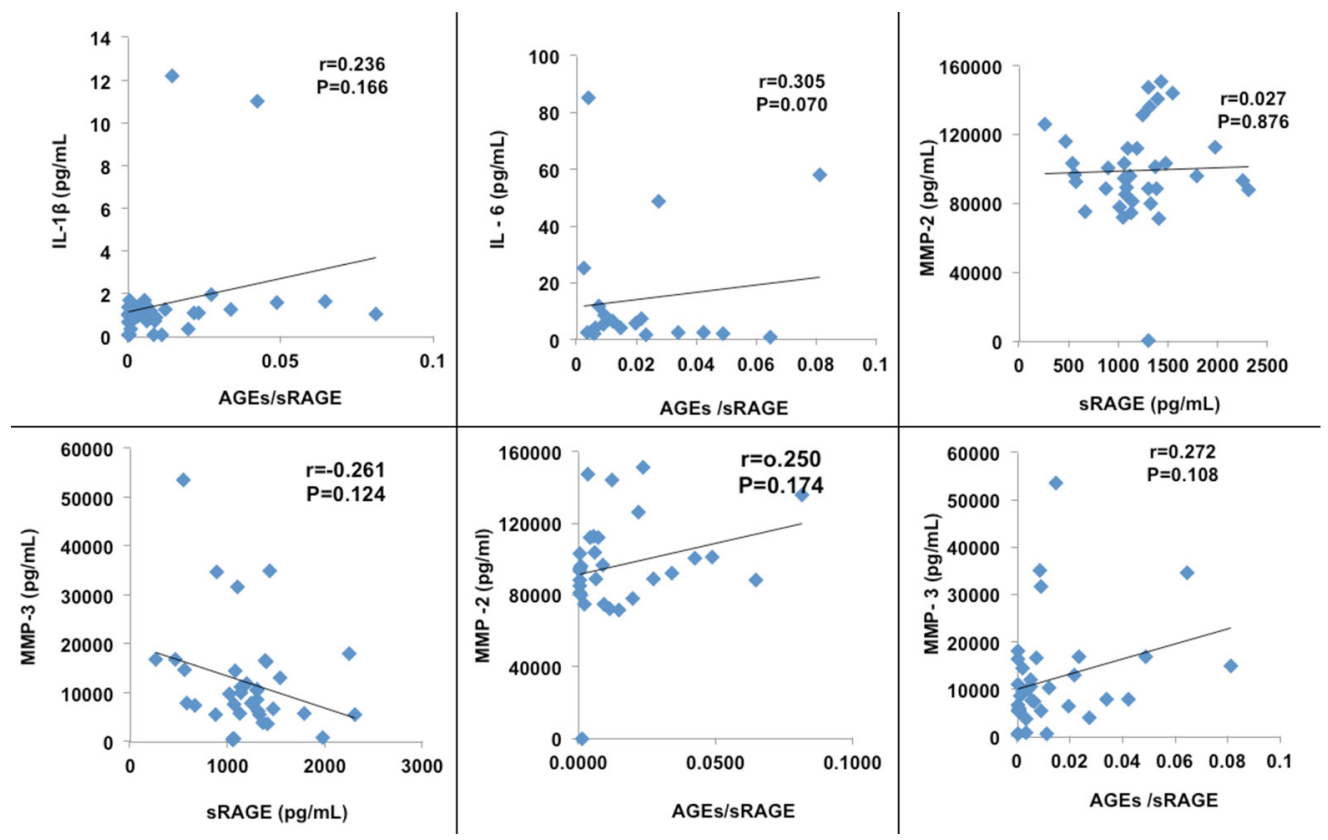

Figure 6. Correlation of AGEs/sRAGE with IL-I $3, \mathrm{IL}-6, \mathrm{MMP}-2$, and MMP-3, and of sRAGE with MMP-2 and -3. IL = interleukin; AGEs = advanced glycation end products; sRAGE = soluble receptor for AGEs; MMP = matrix metalloproteinase. 
Table 2. Correlation coefficients $(n=31)$.

\begin{tabular}{lcc}
\hline Parameter & Pearson correlation & P value \\
\hline IL-1 $\beta$ vs. esRAGE & 0.223 & 0.227 \\
IL-2 vs. esRAGE & 0.291 & 0.113 \\
IL-6 vs. esRAGE & 0.124 & 0.507 \\
TNF- a vs. esRAGE & 0.380 & 0.035 \\
MMP-2 vs. esRAGE & 0.081 & 0.665 \\
MMP-3 vs. esRAGE & 0.007 & 0.969 \\
IL-2 vs. AGEs/sRAGE & 0.568 & 0.001 \\
TNF-a vs. AGEs/sRAGE & 0.277 & 0.131 \\
IL-1 $\beta$ vs. AGEs / esRAGE & 0.315 & 0.084 \\
IL-2 vs. AGEs /esRAGE & 0.371 & 0.040 \\
IL-6 vs. AGEs / esRAGE & 0.495 & 0.005 \\
TNF- $a$ vs. AGEs / esRAGE & 0.159 & 0.392 \\
MMP-2 vs. AGEs / esRAGE & 0.152 & 0.415 \\
MMP-3 vs. AGEs / esRAGE & 0.015 & 0.934 \\
MMP-2 vs. TNF-a & 0.293 & 0.110 \\
MMP-3 vs. TNF-a & 0.017 & 0.927 \\
MMP-2 vs. AGEs & 0.176 & 0.343 \\
MMP-3 vs. AGEs & -0.042 & 0.824 \\
\hline
\end{tabular}

$\mathrm{IL}=$ interleukin; TNF- $\mathrm{a}=$ tumor necrosis factor-alpha; MMP = matrix metalloproteinase; $\mathrm{AGEs}=$ advanced glycation end products; esRAGE = endosecretory soluble receptors for AGEs.
Our hypothesis was that there would be positive correlation between IL-B, IL-2, or IL- 6 and MMP-2 and -3 . There was significant positive correlation of IL-I $\beta$ with MMP-2 and -3 , and a weak positive correlation of IL- 2 with MMPs and IL- 6 with MMP-2.

The data in general show that low levels of sRAGE and high levels of AGEs/sRAGE and AGEs/esRAGE are associated with an increase in the serum levels of cytokines and MMPs. Also, there is an inverse correlation between SRAGE and cytokines or MMP-3, and a positive correlation between AGE/sRAGE or AGE/esRAGE, and cytokines and MMPs. The serum levels of cytokines are positively correlated with MMPs.

In conclusion, the data suggest that low levels of sRAGE, and high levels of AGEs/sRAGE and AGEs/esRAGE, increase the levels of cytokines that in turn increase the levels of MMPs resulting in the formation of TAAs. The data suggest also that the AGE-RAGE axis may be involved in the pathogenesis of TAAs and that low levels of sRAGE and high levels of AGEs, AGES/sRAGE, and AGEs/esRAGE may be new risk factors for TAAs. This new mechanism of development of TAAs may open new avenues for the prevention, regression, and slowing of TAA progression. These findings may also help for

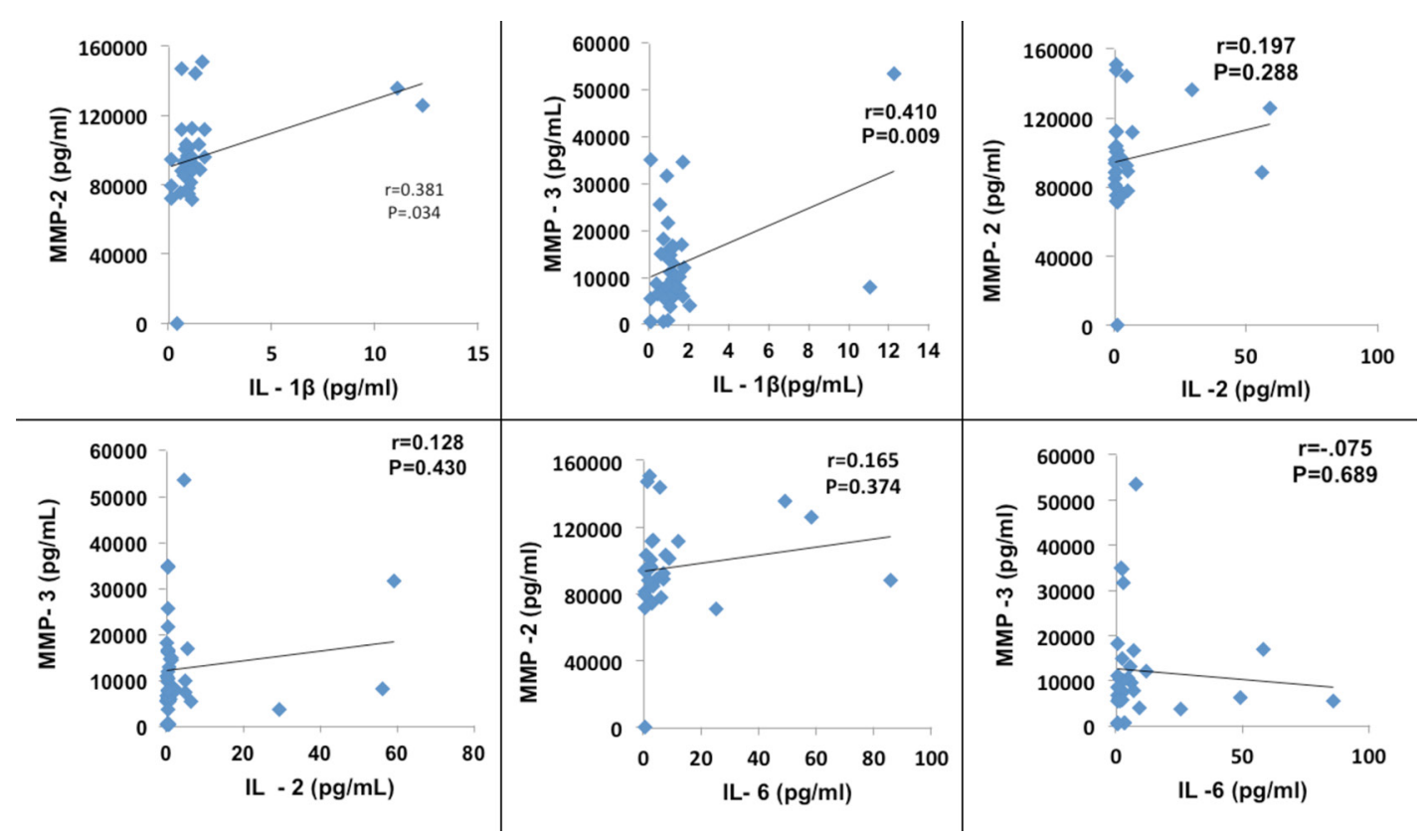

Figure 7. Correlation of IL-Iß, IL-2, and IL-6 with MMP-2 and -3. IL = interleukin; MMP = matrix metalloproteinase. 
screening of population for early detection of TAAs via biomarkers chosen from the pathophysiology herein demonstrated.

\section{Acknowledgments}

This work was supported by the college of Medicine Research Fund of the University of Saskatchewan.

\section{References}

1. Kotze CW, Ahmad IG. Etiology and pathology of aortic aneurysm. In: Grundmann R. Etiology, pathogenesis and pathophysiology of aortic aneurysms and aneurysmal rupture. July 2011, pp. 1-31. DOI: $10.5772 / 18257$

2. DiMusto PD, Upchurch GR. The pathogenesis of abdominal aortic aneurysms. Available at http://admin.vascularweb. org/research/Pages/the-pathogenesis-of-abdominal-aortic-aneurysms.aspx. Accessed on November, 2014.

3. Mountain DJ, Singh M, Menon B, Singh K. Interleukin-1 beta increases expression of activity of matrix metalloproteinase-2 in cardiac microvascular endothelial cells: Role of PKC alpha/beta 1 and MAPKs. Am J Physiol Cell Physiol. 2007;292:C867-C875. DOI: 10.1152/ajpcell.00161.2006

4. Wong W-R, Kossodo S, Kochevar IE. Influence of cytokines on matrix metalloproteinases produced by fibroblasts cultured in monolayer and collagen gells. J Formos Med Assoc. 2001;100:377-382. PMID: 11480246

5. Edsparr K, Speetjens FM, Mulder-Stapel A, Goldfarb RH, Basse PH, Lennermas B, et al. Effects of IL-2 on MMP expression in freshly isolated human NK cells and the IL-2-independent NK cell line YT. J Immunother. 2010;33:475-481. DOI: 10.1097/CJI.0b013e3181d372a0

6. Kusano K, Miyaura C, Inda M, Tamura T, Ito $A$, Nagase $\mathrm{H}$, et al. Regulation of matrix metalloproteinases (MMP-2,-3,-9, and -13) by interleukin- 1 and interleukin- 6 in mouse calvaria: Association of MMP induction with bone reabsorption. Endocrinology. 1998;139:1338-1345. DOI: 10.1210/endo.139.3.5818\#sthash.BIYPY8aH.dpuf

7. Bucala R, Cerami A. Advanced glycosylation: Chemistry, biology, and implications for diabetes and aging. Adv Pharmacol. 1992;23:1-34. DOI: 10.1016/S1054-3589 (08)60961-8
8. Prasad K. Soluble receptor for advanced glycation end products (sRAGE) and cardiovascular disease. Int J Angiol. 2006;15:57-68.

9. Hoffman MA, Drury S, Fu C, Qu W, Taguchi A, Lu Y, et al. RAGE mediates a novel proinflammatory axis: A central surface receptor for S100/ calgranulin polypeptides. Cell. 1999;97:889-901. DOI: 10.1016/S00928674(00)80801-6

10. Lappas M, Permezel M, Rice G. Advanced glycation end products mediate pro-inflammatory actions in human gestational tissues via nuclear factor- $\mathrm{KB}$ and extracellular signal-regulated kinase 1/2. J Endocrinol. 2007;193:269-277. DOI: 10.1677/JOE-06-0081

11. Schmidt AM, Yan SD, Yan SF, Stern DM. The biology of receptor for advanced glycation end products and its ligands. Biochem Biophys Acta. 2000;1498: 99-111. DOI: 10.1016/S0167-4889(00) 00087-2

12. Tam XHL, Shiu SWM, Leng $L$, Bucala $R$, Betteridge DJ, Tan KCB. Enhanced expression of receptor for advanced glycation end-products is associated with low circulating soluble isoforms of the receptors in Type 2 diabetes. Clin Sci. 2011;120:81-89. DOI: 10.1042/CS20100256

13. Yonekura $H$, Yamamoto $Y$, Sakurai $S$, Petrova RG, Abedin MJ, Li H. Novel splice variants of the receptor for advanced glycation end products expressed in human vascular endothelial cells and pericytes, and their putative roles in diabetes-induced vascular injury. Biochem J. 2003;370:1097-1109. DOI: 10.1042/ bj20021371

14. Geroldi D, Falcone C, Emanuele E. Soluble receptor for advanced glycation end products: from disease marker to potential therapeutic target. Current Med Chem. 2006;13:1971-1978. DOI: $10.2174 / 092986706777585013$
15. Siwik DA, Pagano PJ, Colucci WS. Oxidative stress regulates collagen synthesis and matrix metalloproteinase activity in cardiac fibroblasts. Am J Physiol Cell Physiol. 2001;280:C53-C60. PMID: 11121376

16. Sarkar A, Prasad K, Ziganshin BA, Elefteriades JA. Reasons to investigate the soluble receptor for advanced glycation end products (sRAGE) pathway in aortic disease. Aorta (Stamford). 2013:1:210-217. DOI: 10.12945/j.aorta.2013.13-047

17. Fulton RJ, McDade RL, Smith PL, Keinker L J, Kettman JR Jr. Advanced multiplexed analysis with the FlowMetrix system. Clin Chem. 1997;43:1749-1756. PMID: 9299971

18. Martins TB, Pasi BM, Pickering JW, Jaskowski TD, Litwin CM, Hill HR. Determination of cytokine responses using a multiplexed fluorescent microsphere immunoassay. Am J Clin Pathol. 2002;118:346-353. DOI: 10.1309/NOT6-C56B-GXB2-NVFB

19. Juvonen I, Surcel HM, Satta J, Teppo AM, Bloiqu A, Syrialo $H$, et al. Elevated circulating levels of inflammatory cytokines in patients with abdominal aortic aneurysm. Arterioscler Throm Vasc Biol. 1997;17:2843-2847. DOI: 10.1161/01. ATV.17.11.2843

20. Rohde LE, Arroyo LH, Rifai N, Creager MA, Libby $\mathrm{P}$, Ridker PM, et al. Plasma concentrations of interleukin- 6 and abdominal aortic diameter among subjects without aortic dilatation. Arterioscler Throm Vasc Biol. 1999;19:1695-1699. DOI: 10.1161/01. ATV.19.7.1695

21. Jones KG, Brull DJ, Brown LC, Sian M, Greenhalgh RM, Humphries $S E$, et al. Interleukin-6 (IL-6) and the prognosis of abdominal aortic aneurysms. Circulation. 2001;103:2260-2265. DOI: 10.1161/01. CIR.103.18.2260

22. Karapanagiotidis GT, Antonitsis $P$, Charokopos N, Foroulis N, Anastasiadis $\mathrm{K}$, Rouska $\mathrm{E}$, et al. Serum levels of matrix 
metalloproteinases-1, $-2,-3$, and -9 in thoracic diseases and acute myocardial ischemia. J Cardiothorac Surg. 2009;4:59. DOI: 10.1186/1749-8090-4-59

23. Simova J, Skvor J, Reissigova J, Dudra J, Lindner J, Capek P, et al. Serum levels of matrix metalloproteinases 2 and 9 and TGFBR2 gene screening in patients with ascending aortic dilatation. Folia Biologica (Praha). 2013;59:154-161.
24. Nordon IM, Hinchliffe RJ, Holt PJ, Loftus IM, Thompson MM. Review of current theories for abdominal aortic aneurysm pathogenesis. Vascular. 2009;17:253-263. DOI: 10.2310/6670.2009.00046

25. McMillan WE, Pearce WH. Increased plasma levels of metalloproteinase- 9 are associated with abdominal aortic aneurysms. J Vasc Surg. 1999;29:122-127. DOI: 10.1016/ S0741-5214(99)70363-0
Cite this article as: Prasad K, Sarkar A, Zafar MA, Shoker A, Moselhi HE, Tranquilli $M$, Ziganshin BA, Elefteriades JA. Advanced Glycation End Products and its Soluble Receptors in the Pathogenesis of Thoracic Aortic Aneurysm. AORTA (Stamford) 2016;4(1):1-10. DOI: http:// dx.doi.org/10.12945/j.aorta.2015.15.018 